\title{
Gas Adsorption Performance of Organic Thin Films Prepared by Plasma-Assisted Vacuum Evaporation with Polyimide Precursors
}

\author{
Satoru Iwamori ${ }^{1,2, *}$, Ryousuke Sugimoto ${ }^{1}$, Kousuke Osada ${ }^{1}$, Masahiko Kurata $^{1}$, \\ Hiroyuki Matsumoto ${ }^{1,3}$ and Kazutoshi Noda ${ }^{4}$ \\ ${ }^{1}$ Graduate School of Natural Science and Technology, Kanazawa University, \\ Kakuma-machi, Kanazawa 920-1192, Japan \\ ${ }^{2}$ Department of Mechanical Engineering, School of Engineering, Tokai University, \\ 4-1-1 Kitakaname, Hiratsuka, Kanagawa 259-1292, Japan \\ ${ }^{3}$ Corporate Advanced Technology Center, Iwasaki Electric Co., Ltd., \\ 1-20 Fujimicho, Gyoda 361-0021, Japan \\ ${ }^{4}$ Institute for Environmental Management Technology, \\ National Institute of Advanced Industrial Science and Technology (AIST), \\ Onogawa, Tsukuba 305-8569, Japan
}

(Received January 26, 2010; accepted May 13, 2010)

Key words: plasma, vacuum evaporation, quartz crystal microbalance, polyimide

Organic thin films were deposited onto a quartz crystal with pyromellitic dianhydride (PMDA) and oxydianiline (ODA) by vacuum evaporation with or without argon plasma, and adsorption properties on these thin films for volatile organic compounds (VOCs), i.e., acetone, acetaldehyde, toluene, methyl salicylate, and some types of alcohol, were evaluated using a quartz crystal microbalance (QCM), to investigate their potential as gas sensors. The number of adsorbed gas molecules increased with decreasing molecular weight on each organic thin film. Furthermore, the numbers of adsorbed gas molecules on the organic thin films deposited by vacuum evaporation with argon plasma were higher than those on the organic thin films deposited by vacuum evaporation without the plasma. The numbers of adsorbed gas molecules on both the polyimide thin films with and without argon plasma decreased with increasing temperature in the adsorbed-gasmeasuring system.

${ }^{*}$ Corresponding author: e-mail: iwamori@tokai-u.jp 


\section{Introduction}

Polyimides (PIs) have excellent thermal stability, chemical stability and electrical properties. $^{(1,2)}$ PI films have been used as an insulating layer in print circuit boards (PCBs). ${ }^{(3)}$ Vapor deposition polymerization (VDP) of PI has been reported.(4,5) Two types of monomer are evaporated onto the surface of a substrate in a vacuum chamber in VDP.(6) Many types of monomer are used for coating the PI thin films, e.g., Usui et al. reported the characterization of a PI thin film prepared by coevaporation with perylene tetracarboxylic dianhydride and diaminododecane. ${ }^{(7)}$

In addition, the quartz crystal microbalance (QCM) is one of the sensor devices that enable the measurement of nanogram-order changes in mass on the surface of a quartz crystal by the observation of frequency shifts. ${ }^{(8)}$ Therefore, the QCM method can be applied to different gas-sensing systems by selecting the appropriate electrode material to be formed on the quartz crystal as the detection layer. ${ }^{(9)}$

Highly sensitive detection technologies for volatile organic compounds are required. The QCM method has been applied to the detection of gas molecules, ${ }^{(10-14)}$ and it has been one of the effective methods for the detection of volatile organic compounds (VOCs). We have already reported that the adsorbed quantities of water, ethanol and acetone on a sputtered thin film prepared by reactive sputtering with nitrogen were larger than those on a sputtered thin film prepared by sputtering with argon, ${ }^{(15)}$ and $\mathrm{rf}$ power and pressure during the sputtering affect the surface properties, i.e., adhesion, mechanical and tribological properties, of the thin film sputtered with argon. ${ }^{(16)}$ We also report on the adsorption properties of water and VOCs on the sputtered thin films deposited onto the QCM by rf sputtering with a PI target to characterize their surface properties. ${ }^{(17)}$

Furthermore, we have already reported the molecular structures and surface morphologies of the pyromellitic dianhydride (PMDA), oxydianiline (ODA), polyamic acid (PAA) and PI thin films prepared by vacuum evaporation with or without argon plasma. Many minute prominences, whose diameters were $100-2,000 \mathrm{~nm}$ at the base and heights were several hundreds of $\mathrm{nm}$, can be observed at the surface of the PMDA and ODA thin films prepared by vacuum evaporation without Ar plasma. However, these minute prominences cannot be observed at the surface of the PMDA and ODA thin films prepared by vacuum evaporation with Ar plasma. The surface roughness of the PAA and PI thin films prepared by vacuum evaporation decreased owing to the presence of the plasma during the deposition. ${ }^{(18)}$ In addition, all of these organic thin films prepared with the plasma had hydrophilic surfaces compared with those prepared without the plasma. The surface roughness of these thin films has a smaller effect on the wettability than hydrophilic moieties. ${ }^{(18)}$

In this paper, we report on adsorption properties for the VOCs on the organic thin films deposited by vacuum evaporation with or without argon plasma with polyimide precursors, PMDA and ODA, using a quartz crystal microbalance (QCM) method. 


\section{Experimental Procedure}

\subsection{Deposition of organic thin films by vacuum evaporation}

Figure 1 shows a schematic diagram of the vacuum evaporation apparatus equipped with an rf coil. After the PMDA and ODA were separately enclosed into Knudsen cells located $120 \mathrm{~mm}$ under a glass substrate, they were heated by tungsten heaters. A QCM electrode was equipped on the glass substrate. The temperatures of the cells were controlled by proportional integral differential controllers. ${ }^{(19)}$ The substrate was maintained at room temperature during the deposition. Figure 2 shows a reaction scheme of PAA synthesis and conversion to PI. The PAA was synthesized by the PMDA and ODA. The PI was polymerized by the heat treatment of the PAA. After the chamber was evacuated to a pressure of $1.0 \times 10^{-2} \mathrm{~Pa}$, the pressure in the chamber was maintained at $0.3 \mathrm{~Pa}$ by adjusting the main valve installed in the sputtering system. Any gas was not introduced into the chamber for vacuum evaporation. However, argon was introduced into the chamber for plasma-assisted vacuum evaporation. After the cells enclosing PMDA and ODA were heated to 215 and $205^{\circ} \mathrm{C}$, respectively, they were deposited onto the surface of the QCM electrode and formed a PAA thin film. A PI thin film was formed by heating the PAA thin film at $300^{\circ} \mathrm{C}$ for $60 \mathrm{~min}$ in air. ${ }^{(20)}$

The frequency of the power supply was $13.56 \mathrm{MHz}$, and the rf power supplied into the coil was $100 \mathrm{~W}$. The coating thickness of these organic thin films was about $300 \mathrm{~nm}$.

The thickness of these organic thin films was determined by measuring the height between the film and the glass slide with a profilometer, Surfcom 1400A-6 ${ }^{\circledR}$ (Tokyo Seimitu, Inc., Japan).

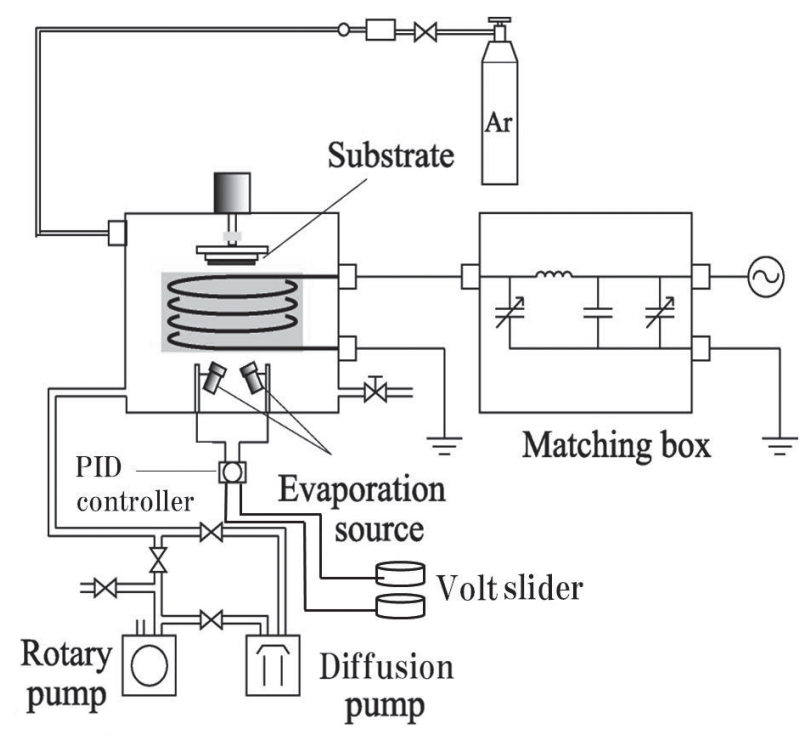

Fig. 1. Schematic diagram of plasma assisted vacuum evaporation system. 

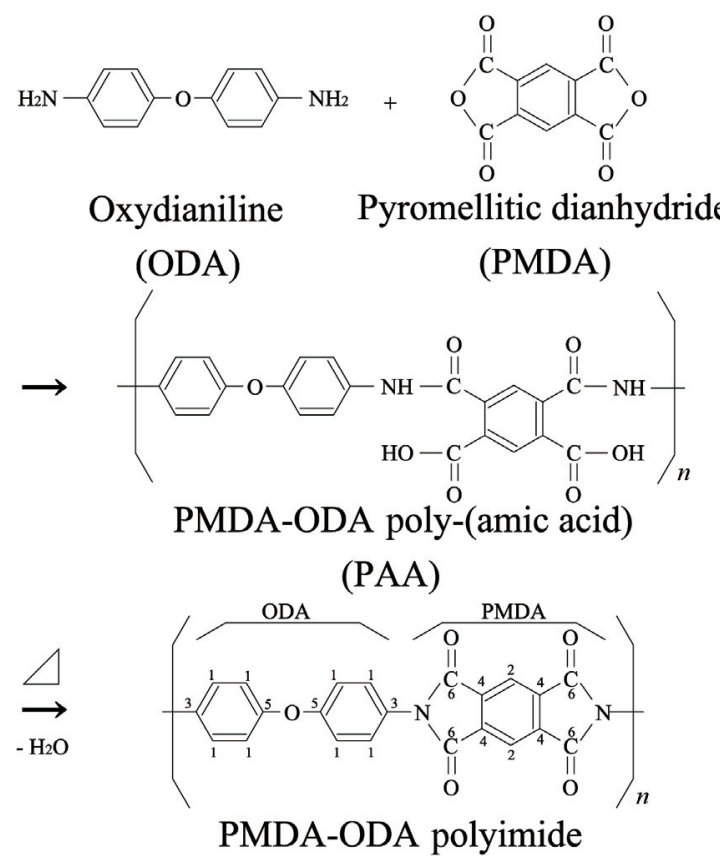

(PI)

Fig. 2. Reaction scheme of polyamic acid (PAA) synthesis and conversion to polyimide (PI). ${ }^{(20)}$

\subsection{Measurent system of gas adsorption}

The adsorption properties of these thin films for acetone, acetaldehyde, toluene, methyl salicylate, and several types of alcohol were evaluated by measuring frequency shifts using the QCM system. An AT-cut QCM device, whose fundamental oscillation frequency is $9 \mathrm{MHz}$, was used in this experiment. ${ }^{(9)}$ After a filter paper soaked in a liquid of these VOCs was placed into a gas washing bottle, dry nitrogen was supplied into the gas washing bottle at $100 \mathrm{~mL} / \mathrm{min}$. The volume of each liquid drop was 100 $\mu \mathrm{L}$. We consider that this volume is sufficient for saturating the response of the QCM system because no significant differences in the QCM oscillating mass could be observed during the measurements of these VOCs. The change in QCM oscillating mass owing to gas adsorption on the organic thin films deposited on the gold electrode of QCM was indicated as a frequency shift. Figure 3 shows a schematic diagram of an adsorbed-gasmeasuring system with QCM. The QCM system was maintained at $20 \pm 1{ }^{\circ} \mathrm{C}$.

A change in frequency was plotted as a frequency shift per gram organic thin film. We have reported the adsorption properties of thin films deposited onto a quartz crystal by rf sputtering with a poly(biphenyltetracarboxylic dianhydride - paraphenylene diamine) (BPDA-PDA) (polyimide) target, and small gas molecules can easily penetrate 




Fig. 3. Schematic diagram of adsorbed-gas-measuring system with QCM.

into the sputtered thin films and can be adsorbed; therefore, the number of adsorbed gas molecules increases. ${ }^{(17)}$ The adsorption properties of these thin films for acetone, acetaldehyde, toluene, methyl salicylate, and several types of alcohol were evaluated. A change in QCM oscillating mass, $\Delta m$, can be calculated using Sauerbrey's equation as ${ }^{(8)}$

$$
\Delta f=-\frac{\Delta m \times F^{2}}{\mathrm{~N} \times S \times r}
$$

where $\Delta f$ is the change in frequency, $\Delta m$ is the change in QCM oscillating mass, $F$ is the fundamental frequency of the QCM, $\mathrm{N}$ is the frequency constant (AT cut is 167 $\mathrm{cm} \cdot \mathrm{kHz}), S$ is the surface area and $r$ is the crystal density $\left(2.65 \mathrm{~g} / \mathrm{cm}^{3}\right)$. In this study, the relationship between $\Delta m$ and $\Delta f$ can be represented as

$$
\Delta m=0.69 \times \Delta f[\mathrm{ng}] .
$$




\section{Results and Discussion}

Figure 4 shows the numbers of adsorbed gas molecules for acetone, acetaldehyde, toluene, and methyl salicylate on these organic thin films deposited by vacuum evaporation with or without argon plasma. The adsorbed quantities of these VOCs are indicated as numbers of gas molecules per gram organic thin film. The number of adsorbed gas molecules increased with decreasing molecular weight on each organic thin film. We consider that adsorption phenomena on these organic thin films deposited by vacuum evaporation with or without argon plasma are similar to those on the sputtered thin films. Furthermore, the numbers of adsorbed gas molecules on the organic thin films deposited by vacuum evaporation with argon plasma were higher than those on the organic thin films deposited by vacuum evaporation without argon plasma. We have reported that the oxygen or nitrogen contents of these organic thin films prepared with Ar plasma slightly decreased compared with those of the organic thin films prepared without Ar plasma; however, the wettability at the surfaces of these organic thin films tends to increase owing to the Ar plasma, and polar moieties are formed by the Ar plasma. ${ }^{(18)}$ VOC molecules may interact with the polar moieties in these organic thin films.

Figure 5 shows the numbers of adsorbed gas molecules for methanol, ethanol, 1-propanol, and 1-butanol on the polymerized organic (polyimide) thin films prepared by vacuum evaporation with or without argon plasma after heat treatment at $300^{\circ} \mathrm{C}$ for $60 \mathrm{~min}$ in air. In both the polymerized organic thin films, the numbers of adsorbed gas

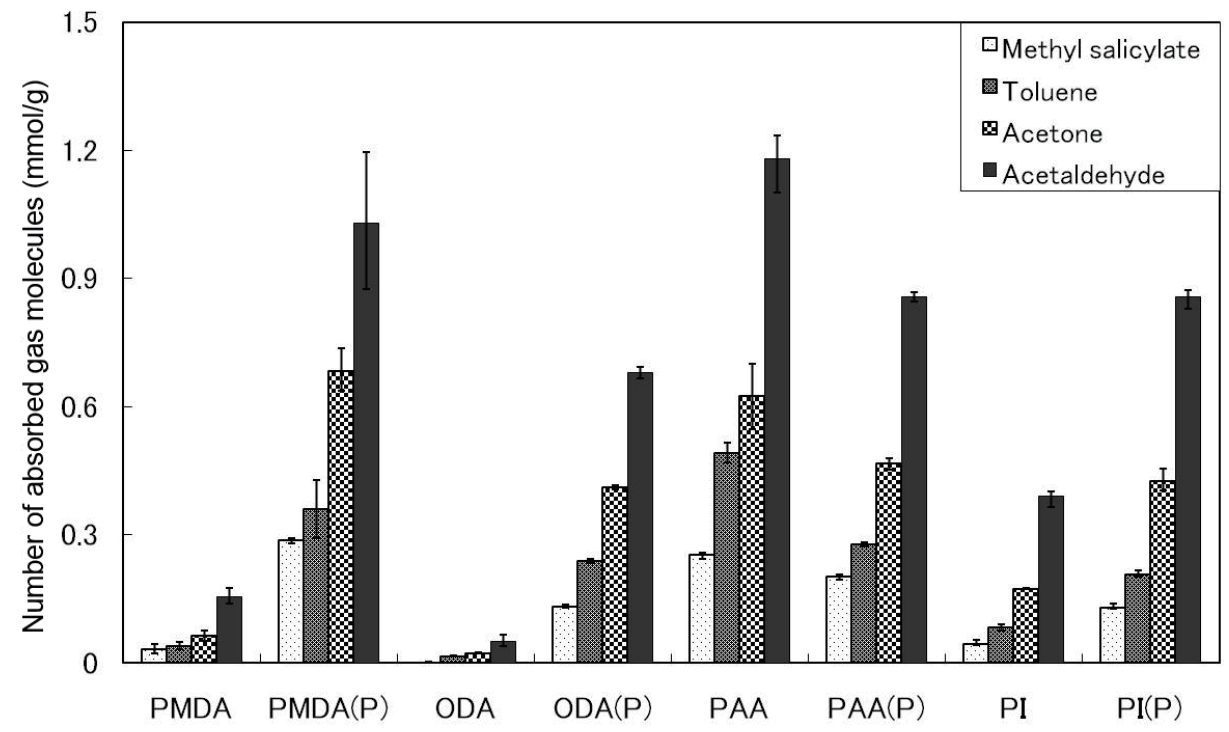

Fig. 4. Numbers of adsorbed gas molecules on the organic thin films deposited by vacuum evaporation with or without argon plasma (P). 




Fig. 5. Numbers of adsorbed gas molecules for alcohols ( $\mathrm{mmol} / \mathrm{g})$.

molecules increased with decreasing molecular weight. In addition, by considering the molar polarization $P_{\mathrm{M}}$ values of methanol, ethanol, 1-propanol, and 1-butanol, the relationship between the molar polarization and the numbers of adsorbed gas molecules for alcohols was determined and is shown in Fig. 6.

The numbers of adsorbed gas molecules increased with decreasing molar polarization. The molar polarization can be calculated using the Clausius-Mosotti equation as ${ }^{(21)}$

$$
P_{\mathrm{M}}=\frac{\varepsilon-1}{\varepsilon+2} \cdot \frac{M}{\rho},
$$

where $P_{\mathrm{M}}$ is the molar polarization, $\varepsilon$ is the dielectric constant, $M$ is the molecular weight, and $\rho$ is the density of these alcohols.

Table 1 shows the dielectric constants, molecular weights and densities of the alcohols. By considering these values and eq. (3), the effect of the molecular weight on the molar polarization was determined to be the largest between those of the three parameters. We consider that this is one of the reasons why the numbers of adsorbed gas molecules increased with decreasing molar polarization as well as molecular weight.

Figure 7 shows the numbers of adsorbed methanol molecules at various temperatures in the adsorbed-gas-measuring system. The numbers of adsorbed methanol molecules on both the polymerized organic thin films with and without argon plasma decreased with increasing temperature.

Figure 8 shows the numbers of adsorbed toluene molecules at various temperatures in the adsorbed-gas-measuring system. The numbers of adsorbed toluene molecules on 


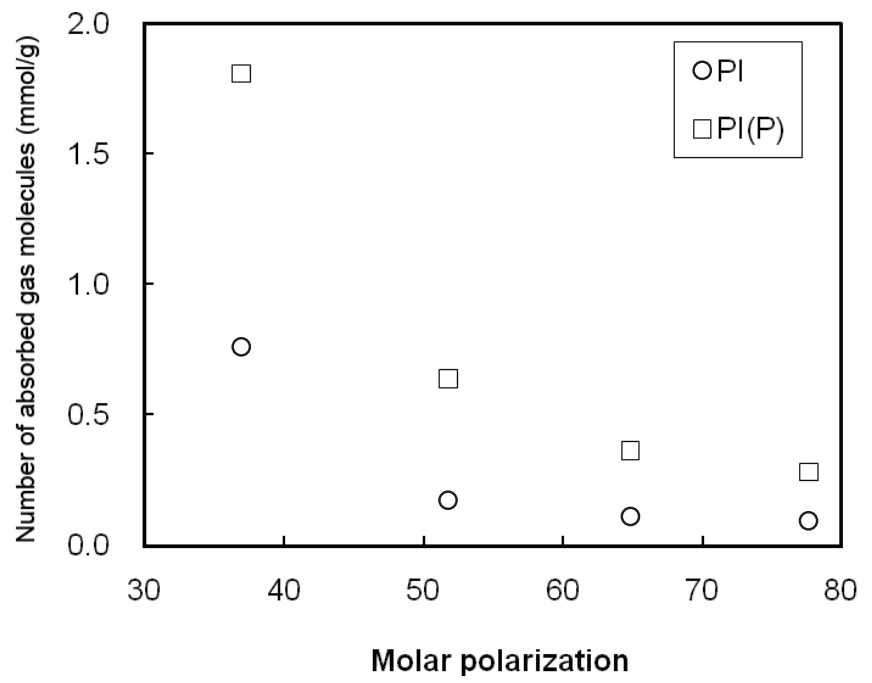

Fig. 6. Relationship between molar polarization and numbers of adsorbed gas molecules for alcohols.

Table 1

Dielectric constants, molecular weights and densities of these alcohols.

\begin{tabular}{lcccc}
\hline & Methanol & Ethanol & 1-Propanol & 1-Butanol \\
\hline$\varepsilon$ & 33 & 25 & 21 & 18 \\
$M$ & 32 & 46 & 60 & 74 \\
$\rho$ & 0.792 & 0.789 & 0.804 & 0.81 \\
\hline
\end{tabular}



Fig. 7. Numbers of adsorbed gas molecules for methanol at various temperatures. 


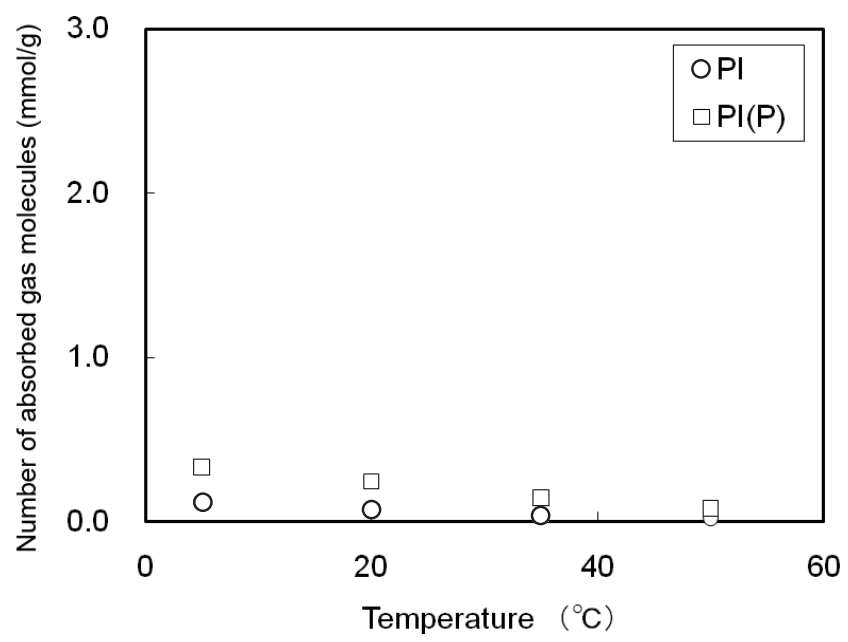

Fig. 8. Numbers of adsorbed gas molecules for toluene at various temperatures.

both the polymerized organic thin films with and without argon plasma also decreased with increasing temperature. We consider that the adsorbed gas molecules would be eliminated and decreased in number owing to the activation of the adsorbed gas molecules on these organic thin films at high temperatures. Methanol is a polar solvent, and toluene is a nonpolar solvent. This tendency does not depend on the polarity of the adsorbed gas molecules.

\section{Conclusion}

We investigated the adsorption properties of organic thin films deposited onto a quartz crystal with pyromellitic dianhydride (PMDA) and oxydianiline (ODA) by vacuum evaporation with or without argon plasma. The numbers of adsorbed gas molecules on the organic thin films deposited by vacuum evaporation with argon plasma were higher than those on the organic thin films deposited by vacuum evaporation without argon plasma. These organic thin films have high sensitivities for low-molecular-weight VOCs and low-molar-polarization VOCs. Furthermore, the number of adsorbed VOC gas molecules decreased with increasing temperature in the adsorbed-gas-measuring system. The decrease in the number of adsorbed gas molecules at high temperatures would be due to the activation of the adsorbed gas molecules on these organic thin films.

\section{Acknowledgement}

This work was partially supported by a Grant-in-Aid for Scientific Research C (No. 21560744) from the Ministry of Education, Culture, Sports, Science and Technology of Japan. 


\section{References}

1 M. K. Ghosh and K. L. Mittal (eds.): Polyimides: Fundamentals and Applications (Marcel Dekker, New York, 1996).

2 K. L. Mittal (ed.): Polyimides and Other High Temperature Polymers 1 (VSP, Utrecht, 2001).

3 Y. Nakamura, Y. Suzuki and Y. Watanabe: Thin Solid Films 290/291 (1996) 367.

4 R. Salem, F. O. Sequed, J. Duran and W. Y. J. Lee: J. Vac. Sci. Technol. A 4 (1986) 369.

5 Y. Takahashi, M. Iijima, K. Inagawa and A. Itoh: J. Vac. Sci. Technol., A 5 (1987) 2253.

6 Y. Takahashi, K. Matsuzaki, M. Iijima, E. Fukada, S. Tsukahara, Y. Murakami and A. Maesono: Jpn. J. Appl. Phys. 32 (1993) L875.

7 H. Usui, K. Hibi, M. Watanabe and K. Tanaka: Int. Symp. Solid State Devices and Materials F-8-6 (2004) 858.

8 G. Z. Sauerbrey: Z. Phys. 155 (1959) 206.

9 K. Noda, R. Naganawa, Y. Kanekiyo and H. Tao: Chem. Lett. 32 (2003) 1128.

10 V. Syritski, J. Reut, A. Öpik and K. Idla: Synth. Met. 102 (1999) 1326.

11 K. Hirayama, Y. Sakai, K. Kameoka, K. Noda and R. Naganawa: Sens. Actuators, B 86 (2002) 20.

12 K. Noda, K. Matsunobu, H. Aizawa, S. Kurosawa, R. Naganawa and H. Tao: IEEJ Trans. SM 125 (2005) 50 (in Japanese).

13 P. G. Su and Y. P. Chang: Sens. Actuators, B 129 (2008) 915.

14 Z. Ying, Y. Jiang, X. Du, G. Xie, J. Yu and H. Tai: Eur. Polym. J. 44 (2008) 1157.

15 A. Uemura, S. Yano, S. Iwamori and K. Noda: J. Mater. Sci. Soc. Jpn. 45 (2008) 143 (in Japanese).

16 A. Uemura, T. Tanabe and S. Iwamori: IEEJ Trans. FM 130 (2010) 147 (in Japanese).

17 S. Iwamori, S. Yano, R. Sugimoto, A. Uemura, H. Matsumoto and K. Noda: Jpn. J. Appl. Phys. 49 (2010) 04DK18.

18 R. Sugimoto, K. Osada, M. Kurata, H. Matsumoto, S. Iwamori and K. Noda: J. Vac. Soc. Jpn. 53 (2010) 176 (in Japanese).

19 M. Watanabe, C. Arai, K. Tanaka and H. Usui: IEICE. OME2000-48 (2000) 33 (in Japanese).

20 R. Dutt, Y. Takahashi and M. Iijima: Jpn. J. Appl. Phys. 38 (1999) L687.

21 H. El-Kashef: Opt. Mater. 10 (1998) 207. 